Trab-780-01 (7 páginas)

PROVA GRÁFICA

\title{
EFEITO DO AIB NO ENRAIZAMENTO DE MINIESTACAS E MICROESTACAS DE CLONES DE Eucalyptus grandis W. Hill ex Maiden ${ }^{1}$
}

\author{
Miranda Titon², Aloisio Xavier ${ }^{3}$, Wagner Campos Otoni ${ }^{4}$ e Geraldo Gonçalves dos Reis ${ }^{3}$
}

\begin{abstract}
RESUMO - O objetivo do presente estudo foi avaliar o efeito da aplicação do regulador de crescimento AIB ( 0 , $1.000,2.000$ e $4.000 \mathrm{mg} / \mathrm{l}$ ) na sobrevivência, no enraizamento e no vigor das microestacas e miniestacas de quatro clones de Eucalyptus grandis. Com relação às características de sobrevivência na saída da casa de vegetação, ao enraizamento na saída da casa de sombra e à sobrevivência das mudas aos 50 dias de idade, observou-se diversidade de resposta dos clones em relação às dosagens do AIB. Entretanto não foi verificado efeito do AIB nas características altura e diâmetro do colo, tanto na microestaquia como na miniestaquia, dos quatro clones. Constatou-se aumento nos índices de enraizamento e de sobrevivência das miniestacas nas dosagens de 1.000 e $2.000 \mathrm{mg} / \mathrm{l}$, na maioria dos clones. Não foi observado efeito no enraizamento e na sobrevivência das microestacas, entretanto ocorreram valores iguais ou superiores aos obtidos na miniestaquia, o que indica maior vigor das microestacas em relação às miniestacas.
\end{abstract}

Palavras-chave: Miniestaquia, microestaquia e propagação vegetativa.

\section{MINICUTTINGS AND MICROCUTTINGS ROOTING OF Eucalyptus grandis W. Hill ex Maiden CLONES AS AFFECTED BY IBA}

\begin{abstract}
Four Eucalyptus grandis clones were used to evaluate the effect of IBA application (0, 1000, 2000 and $4000 \mathrm{mg} / \mathrm{l}$ ) on the survival, rooting and vigor of microcuttings and minicuttings. Regarding greenhouse exit survival and shade house exit rooting and survival at 50 days of age microcuttings and minicuttings, diverse clone responses were observed in relation to IBA dosages. However, for all the tested clones no growth regulator effect was observed on height and root collar diameter for both microcutting and minicutting techniques. Increased rooting indexes and survival of the minicutting were observed in the dosages of 1000 and $2000 \mathrm{mg} / \mathrm{l} I B A$ in most of the clones. In general, no effect was observed on rooting and survival of the microcuttings; however similar or higher values to those obtained by minicutting technique were observed suggesting greater microcutting vigor, as compared to the minicutting.
\end{abstract}

Key words: Minicutting technique, microcutting technique and vegetative propagation.

\section{INTRODUÇÃO}

O desenvolvimento da clonagem de espécies e híbridos do gênero Eucalyptus por meio do enraizamento de estacas foi o marco inicial para a propagação vegetativa assumir posição de destaque e despertar o interesse das empresas e pesquisadores, com conseqüente busca de aprimoramentos e inovações tecnológicas.
$\mathrm{O}$ aprimoramento no enraizamento de estacas tem sido conseguido, especialmente, com o desenvolvimento das técnicas da microestaquia (Assis et al., 1992; Xavier \& Comério, 1996) e da miniestaquia (Xavier \& Wendling, 1998; Wendling, 1999; Wendling et al., 2000a), que possibilitaram consideráveis ganhos decorrentes, principalmente, do aumento dos índices de enraizamento e da redução do tempo para formação da muda.

1 Recebido para publicação em 21.9.2001.

Aceito para publicação em 19.2.2003.

2 Engenheira Florestal, M.S. ${ }^{3}$ Professor do Departamento de Engenharia Florestal da Universidade Federal de Viçosa - UFV, 36571-000 Viçosa-MG; ${ }^{4}$ Professor do Departamento de Biologia Vegetal da UFV. 
Aplicações exógenas de auxina proporcionam maior porcentagem, velocidade, qualidade e uniformidade de enraizamento (Hartmann et al., 1997). Dentre as auxinas mais conhecidas e utilizadas no enraizamento de estacas, têm-se o ácido indolacético (AIA), o ácido indol-butírico (AIB), o ácido naftaleno acético (ANA) e o 2,4-diclorofenoxiacético (2,4-D) (Blazich, 1987). As concentrações do produto ativo variam com a espécie (Wilson, 1994b), o clone (Chung \& Lee, 1994), o estado de maturação do propágulo (Gomes, 1987) e a forma de aplicação, que pode ser via líquido ou via talco (Blazich, 1987).

$\mathrm{Na}$ propagação vegetativa de Eucalyptus por estaquia, o regulador de crescimento de maior uso tem sido o AIB, com melhores resultados apresentados em concentrações de 6.000 a $8.000 \mathrm{mg} / \mathrm{l}$ (Wilson, 1994a). No entanto, a utilização das técnicas de microestaquia e de miniestaquia tem levado ao uso de concentrações mais baixas de AIB e, em alguns casos, até a sua supressão. Para a microestaquia, o uso de reguladores de crescimento não tem sido indicado (Assis et al., 1992; Xavier \& Comério, 1996; Assis, 1997), ao passo que dosagens entre 1.000 e $3.000 \mathrm{mg} / \mathrm{l}$ de AIB incrementaram as taxas de sobrevivência e de enraizamento de miniestacas (Wendling et al., 2000b).

Considerando a importância da microestaquia e da miniestaquia na clonagem de Eucalyptus e a necessidade de maiores informações sobre a utilização de reguladores de crescimento nessas técnicas, objetivou-se avaliar o efeito da aplicação de dosagens de AIB $(0,1.000,2.000$ e $4.000 \mathrm{mg} / \mathrm{l})$ na sobrevivência, no enraizamento e no vigor de microestacas e miniestacas de quatro clones de Eucalyptus grandis.

\section{MATERIAL E MÉTODOS}

Foram utilizados quatro clones de Eucalyptus provenientes da Empresa Celulose Nipo-Brasileira S.A. (CENIBRA), localizada no município de Belo Oriente$\mathrm{MG}$, sendo dois clones de Eucalyptus grandis $(\mathrm{CC} 1 \mathrm{e}$ CC8) e dois de híbridos de Eucalyptus grandis - Rio Claro-SP (CC11 e CC12).

As microcepas utilizadas na formação do jardim microclonal foram provenientes de mudas rejuvenescidas por meio da micropropagação e na composição do jardim miniclonal foram utilizadas minicepas obtidas pelo enraizamento de miniestacas oriundas das brotações de plantas propagadas pelo método da estaquia convencional, conforme descrito por Titon (2001).

R. Árvore, Viçosa-MG, v.27, n.1, p.1-7, 2003
Os jardins clonais foram instalados no viveiro florestal da CENIBRA, em Belo Oriente-MG, sendo cobertos com plásticos transparentes e constituídos pelos clones em estudo. Como recipientes para as microcepas e minicepas foram utilizados tubetes de plástico de $55 \mathrm{~cm}^{3}$, contendo substrato constituído de partes iguais de vermiculita de granulometria média e casca de arroz carbonizada. O sistema de manejo adotado no jardim clonal foi composto por bandejas de aço inoxidável, sobre as quais permaneceram as microcepas e minicepas, acondicionadas em tubetes dispostos em bandejas de plástico rígido. A irrigação e a nutrição mineral foram efetuadas por um sistema automatizado de fertirrigação por inundação, conforme detalhado por Titon (2001).

Para enraizamento e formação das mudas, as microestacas e miniestacas, com dimensões variando de 4 a $6 \mathrm{~cm}$ de tamanho, foram coletadas nos jardins clonais, colocadas para enraizamento em casa de vegetação (permanência de 25 dias), depois para aclimatação em casa de sombra (permanência de oito dias) e posteriormente para rustificação a pleno sol, até completarem 50 dias. A nutrição mineral seguiu os procedimentos descritos por Titon (2001).

Foram utilizadas quatro concentrações do regulador de crescimento $\operatorname{AIB}(0,1.000,2.000$ e $4.000 \mathrm{mg} / \mathrm{l})$, via líquido, dissolvido em hidróxido de sódio $(\mathrm{NaOH})$ a $1 \mathrm{~mol} / \mathrm{l}$ e diluído com água destilada. As microestacas e miniestacas tiveram suas bases mergulhadas na solução de regulador por um período de 10 segundos, antes de serem estaqueadas no substrato.

O experimento foi conduzido no período de setembro a dezembro de 2000 , tendo sido avaliadas a sobrevivência das microestacas e das miniestacas na saída da casa de vegetação; a porcentagem de enraizamento das microestacas e das miniestacas na saída da casa de sombra; e a sobrevivência, a altura e o diâmetro do colo das mudas aos 50 dias de idade.

$\mathrm{O}$ delineamento experimental utilizado foi o inteiramente casualizado, em arranjo fatorial $2 \times 4 \times 4$, constituído de duas técnicas (microestaquia e miniestaquia), quatro dosagens de AIB e quatro clones ( $\mathrm{CC} 1$, $\mathrm{CC} 8, \mathrm{CC} 11$ e CC12), em seis repetições e parcelas compostas de oito plantas.

\section{RESULTADOS E DISCUSSÃO}

As interações "AIB x Técnica", "AIB x Clone" e "Clone x Técnica" apresentaram efeito significativo, pelo 
teste $\mathrm{F}(\mathrm{P}<0,05)$, para algumas características, o que indica a existência de respostas diferenciadas dos clones em relação às técnicas e às dosagens utilizadas (Quadro 1).

Nota-se (Figura 1) que na microestaquia a utilização de AIB, em geral, não influenciou a sobrevivência das microestacas na saída da casa de vegetação. Exceção fazse aos clones CC8 e CC11, em que a dosagem de $4.000 \mathrm{mg} / 1$ apresentou decréscimo dos porcentuais, provavelmente pelo fato de a concentração do regulador de crescimento utilizado apresentar efeitos indesejáveis ao enraizamento. Esses resultados estão de acordo com Xavier \& Comério (1996) e ASSIS et al. (1992), segundo os quais a utilização de regulador de crescimento não é recomendada para microestaquia, podendo, às vezes, ter efeito negativo.

No caso da miniestaquia, para os clones $\mathrm{CC} 1 \mathrm{e}$ CC12, não foi observada variação de resposta no porcentual de sobrevivência na saída da casa de vegetação em relação às dosagens de AIB utilizadas, apresentando comportamento semelhante ao da microestaquia. No entanto, nos clones CC8 e CC11 a aplicação de AIB proporcionou decréscimo nos porcentuais a partir da dosagem de $2.000 \mathrm{mg} / 1$. Os resultados de sobrevivência na saída da casa de vegetação obtidos na miniestaquia concordam com Wendling et al. (2000b), que constataram que os melhores índices de sobrevivência de miniestacas foram obtidos com dosagens de AIB variando entre 1.000 e $3.000 \mathrm{mg} / \mathrm{l}$.

O enraizamento das microestacas e das miniestacas na saída da casa de sombra está representado na Figura 2. Para os clones CC8 e CC12, não foram observadas grandes variações na porcentagem de enraizamento das microestacas e miniestacas nas dosagens de 0 a $2.000 \mathrm{mg} / 1 \mathrm{de}$ AIB. O clone $\mathrm{CC} 1$, na dosagem de $2.000 \mathrm{mg} / \mathrm{l}$, apresentou índices de enraizamento na saída da casa de sombra superiores a 12,5 e $16,1 \%$ na microestaquia e miniestaquia, respectivamente, em detrimento da não-aplicação de AIB. Já para o clone CC11, a partir da dosagem de $1.000 \mathrm{mg} / \mathrm{l}$ observou-se queda nos porcentuais, sendo esta mais acentuada na miniestaquia. A dosagem de $4.000 \mathrm{mg} / \mathrm{l}$ apresentou os menores índices de enraizamento, sendo estes mais evidenciados para os clones CC8 e CC11, o que demonstra maior sensibilidade desses clones ao AIB.

Quadro 1 - Resultados da análise de variância referentes à sobrevivência na saída da casa de vegetação (SOBSCV), ao enraizamento na saída da casa de sombra (ENRSCS) e à sobrevivência (SOB50), à altura (ALT50) e ao diâmetro do colo (DC50) das mudas aos 50 dias de idade, com a aplicação de AIB (AIB) em quatro clones de Eucalyptus grandis (Clo) propagados por miniestaquia e microestaquia (Tec)

Table 1 - Variance analysis for survival at greenhouse exit (SOBSCV); rooting at shade house exit (ENRSCS); and survival (SOB50), height (ALT50) and root collar diameter (DC50) of 50 days of age microcuttings and minicuttings, as affected by IBA application on four Eucalyptus grandis clones (Clo), propagated by microcutting and minicutting techniques (Tec)

\begin{tabular}{|c|c|c|c|c|c|c|}
\hline \multirow[b]{2}{*}{ FV } & \multirow[b]{2}{*}{ GL } & \multicolumn{5}{|c|}{ Quadrado Médio } \\
\hline & & $\begin{array}{c}\text { SOBSCV }^{1 /} \\
(\%)\end{array}$ & $\begin{array}{c}\text { ENRSCS }^{1 /} \\
(\%)\end{array}$ & $\begin{array}{c}\mathrm{SOB}^{1 /} \\
(\%)\end{array}$ & $\begin{array}{c}\text { ALT50 } \\
(\mathrm{cm})\end{array}$ & $\begin{array}{l}\text { DC50 } \\
(\mathrm{mm})\end{array}$ \\
\hline Clone (Clo) & 3 & $0,3084 * *$ & $0,8342 * *$ & $1,1056^{* *}$ & $1059,14^{* *}$ & $6,7057 * *$ \\
\hline Técnica (Tec) & 1 & $0,1980 * *$ & $0,5244 * *$ & $1,9362 * *$ & $165,56^{* *}$ & $7,0022 * *$ \\
\hline Dosagem AIB (AIB) & 3 & $0,3484 * *$ & $0,6463 * *$ & $0,3806^{* *}$ & $4,6646^{\mathrm{ns}}$ & $0,4514^{\mathrm{ns}}$ \\
\hline $\mathrm{AIB} * \mathrm{Tec}$ & 3 & $0,0738 * *$ & $0,1131^{*}$ & $0,1129^{\mathrm{ns}}$ & $3,5946^{\mathrm{ns}}$ & $1,3050^{\mathrm{ns}}$ \\
\hline $\mathrm{AIB} *$ Clo & 9 & $0,1577 * *$ & $0,2455^{* *}$ & $0,2434 * *$ & $3,2456^{\mathrm{ns}}$ & $0,9584^{\mathrm{ns}}$ \\
\hline Clo $*$ Tec & 3 & $0,0853 * *$ & $0,1089 *$ & $0,0991^{\mathrm{ns}}$ & $17,472 *$ & $1,6036^{\mathrm{ns}}$ \\
\hline $\mathrm{AIB} * \mathrm{Clo} * \mathrm{Tec}$ & 9 & $0,0223^{\mathrm{ns}}$ & $0,0178^{\mathrm{ns}}$ & $0,0343^{\mathrm{ns}}$ & $3,0148^{\mathrm{ns}}$ & $0,7369^{\mathrm{ns}}$ \\
\hline Resíduo & 160 & 0,0188 & 0,0425 & 0,0564 & 4,8653 & 0,0335 \\
\hline Média Geral & - & 96,29 & 91,04 & 85,81 & 13,31 & 2,00 \\
\hline $\mathrm{CV}_{\exp }(\%)$ & - & 9,18 & 14,83 & 18,37 & 16,57 & 45,96 \\
\hline
\end{tabular}

$* \mathrm{e}^{* *}=$ significativos a 5 e $1 \%$ de probabilidade, respectivamente, pelo teste $\mathrm{F}$.

$\mathrm{ns}=$ não-significativo a $5 \%$ de probabilidade, pelo teste $\mathrm{F}$.

${ }^{1 /}$ dados transformados em arco-seno $\sqrt{x / 100}$, em virtude de não apresentarem normalidade pelo teste de Lilliefors. 

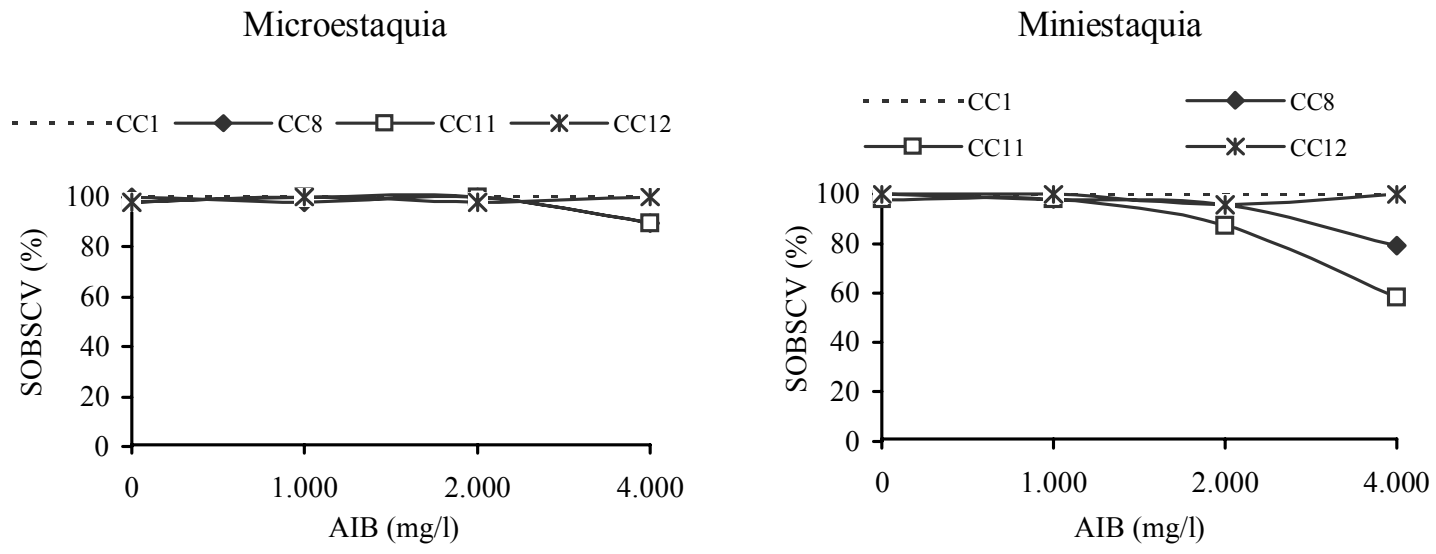

Figura 1 - Médias da sobrevivência das microestacas e miniestacas de quatro clones de Eucalyptus grandis na saída da casa de vegetação (SOBSCV), em resposta à aplicação de AIB $(0,1.000,2.000$ e $4.000 \mathrm{mg} / \mathrm{l}$ ).

Figure 1 - Survival average of microcuttings and minicuttings of four Eucalyptus grandis clones at greenhouse exit (SOBSCV), in response to IBA application (0, 1000, 2000 and $4000 \mathrm{mg} / \mathrm{l})$.
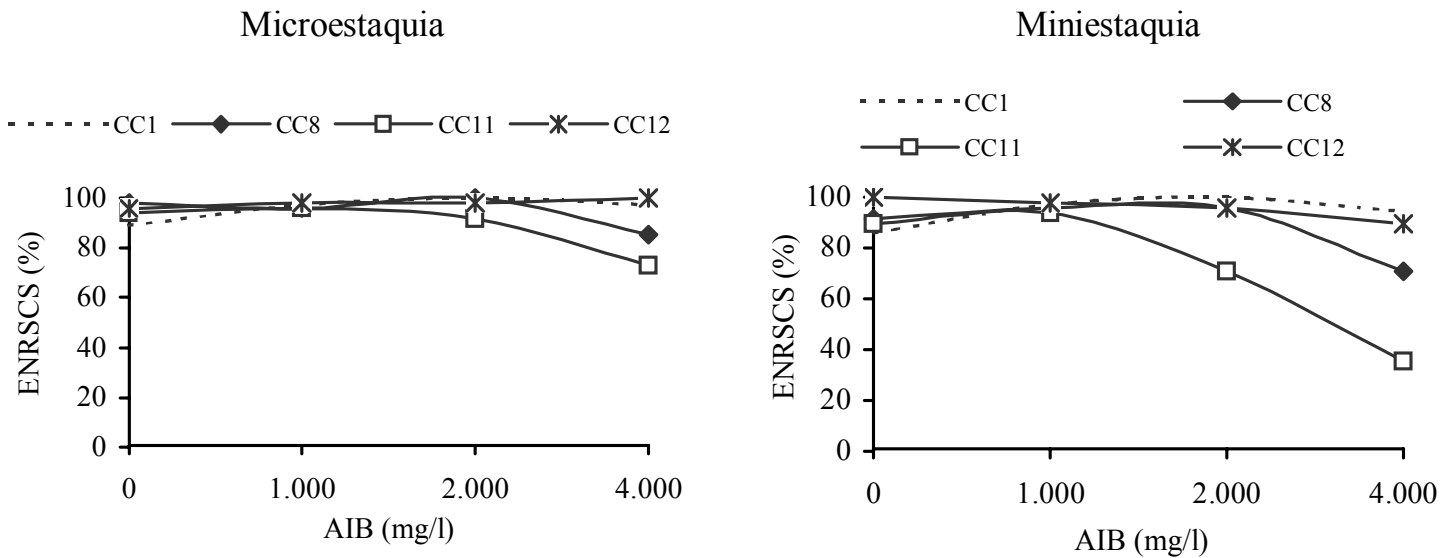

Figura 2 - Médias do enraizamento das microestacas e miniestacas de quatro clones de Eucalyptus grandis na saída da casa de sombra (ENRSCS), em resposta à aplicação de AIB (0, 1.000, 2.000 e $4.000 \mathrm{mg} / \mathrm{l})$.

Figure 2-Rooting average of microcuttings and minicuttings of four Eucalyptus grandis clones at shade house exit (ENRSCS), in response to IBA application $(0,1000,2000$ and $4000 \mathrm{mg} / \mathrm{l})$.

Quanto à sobrevivência das mudas aos 50 dias, o comportamento apresentado pelos clones na microestaquia foi bastante semelhante ao comportamento do enraizamento na saída da casa de sombra. Já na miniestaquia houve maiores variações entre as dosagens e decréscimos mais elevados nos porcentuais de sobrevivência (Figura 3). Para o clone CC1, observou-se superioridade de sobrevivência nas dosagens de 1.000, 2.000 e 4.000 mg/l em relação à não-aplicação de AIB. Considerando a dosagem de $1.000 \mathrm{mg} / \mathrm{l}$, os índices apresentados foram 16,7 e 19,2\% superiores aos da nãoaplicação de AIB, na microestaquia e miniestaquia, respectivamente.

R. Árvore, Viçosa-MG, v.27, n.1, p.1-7, 2003
Na microestaquia, dosagens de 0 e $2.000 \mathrm{mg} / \mathrm{l} \mathrm{de}$ AIB não apresentaram resultados diferenciados para os clones CC8 e CC11, sendo, a partir dessa dosagem, observada queda nos porcentuais de sobrevivência das microestacas. Na miniestaquia, o clone CC8 exibiu maior sobrevivência na dosagem de $2.000 \mathrm{mg} / \mathrm{l}$, o que significa acréscimo de 17,8\% em relação à não-aplicação de AIB. Para o clone CC11, as dosagens de 0 e $1.000 \mathrm{mg} / 1$ foram as melhores, tendo sido observada também queda bastante alta nos índices, a partir dessa dosagem. Para o clone $\mathrm{CC} 12$, à semelhança dos resultados obtidos na saída da casa de sombra, a utilização de AIB não teve efeito significativo na microestaquia. $\mathrm{Na}$ miniestaquia, no 
entanto, observou-se decréscimo nos porcentuais de sobrevivência com o aumento das dosagens de AIB.

A expectativa inicial era de que a aplicação de AIB não promovesse efeito positivo na microestaquia, o que concorda com os trabalhos de Xavier \& Comério (1996) e Assis et al. (1992). No entanto, os resultados obtidos indicaram efeito positivo da aplicação de AIB sobre o clone $\mathrm{CC} 1$. Quanto à miniestaquia, esperava-se, à semelhança dos resultados obtidos por Wendling et al. (2000b), aumento nos índices de enraizamento e sobrevivência com a aplicação de AIB, os quais foram confirmados pelos clones $\mathrm{CC} 1, \mathrm{CC} 8$ e CC11, nas dosagens de 0 a $2.000 \mathrm{mg} / \mathrm{l}$ (Figura 2).

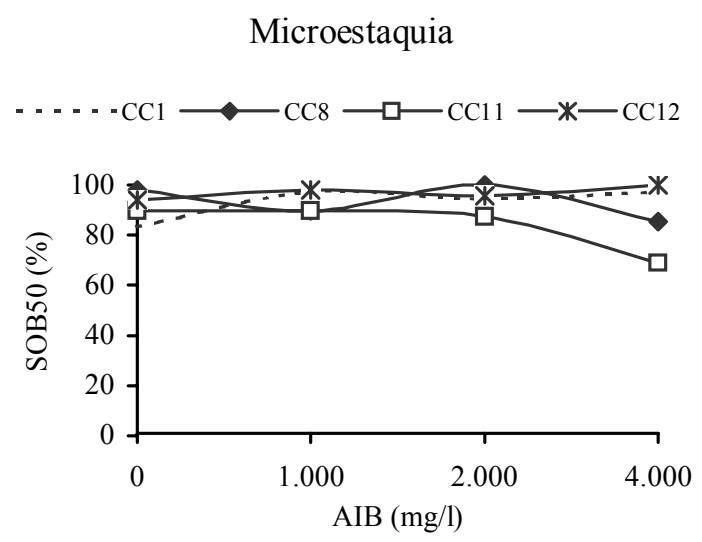

Quanto à comparação da microestaquia e da miniestaquia, o enraizamento das microestacas foi menos influenciado pelo AIB, o que aponta o seu maior vigor vegetativo em relação ao das miniestacas. Essa diversidade de resposta dos clones em relação à utilização de AIB pode estar associada a condições de maturação do material (Wilson, 1994b; Kamlesh et al., 1995), diferenças do material genético (Wilson, 1994b; Chung \& Lee, 1994), condições ambientais, entre outros fatores.

Nas Figuras 4 e 5 estão apresentados os resultados do crescimento em altura e diâmetro do colo das mudas aos 50 dias de idade, na microestaquia e miniestaquia, em resposta à aplicação de AIB. Notou-se, nas duas

Figura 3 - Médias da sobrevivência das mudas de quatro clones de Eucalyptus grandis aos 50 dias de idade (SOB50), em resposta à aplicação de AIB $(0,1.000,2.000$ e $4.000 \mathrm{mg} / \mathrm{l})$.

Figure 3 - Survival average of the microcuttings and minicuttings of four Eucalyptus grandis clones at 50 days of age (SOB50), in response to IBA application (0, 1000, 2000 and $4000 \mathrm{mg} / \mathrm{l})$.
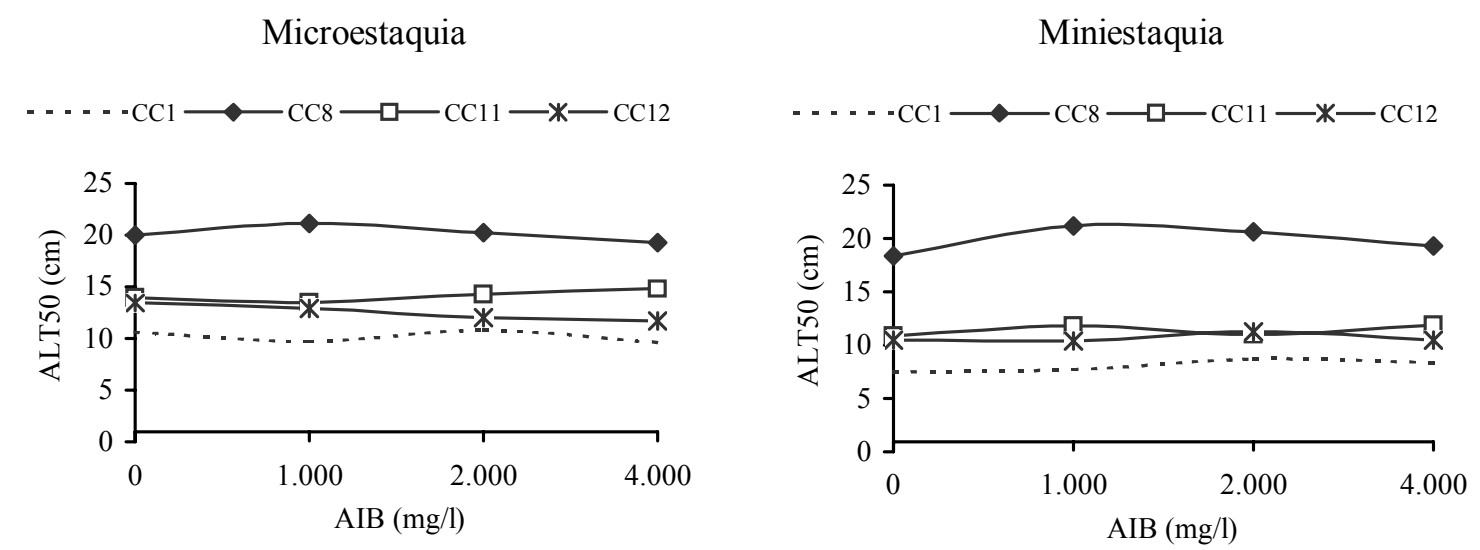

Figura 4 - Médias da altura das mudas de quatro clones de Eucalyptus grandis aos 50 dias de idade (ALT50), em resposta à aplicação de AIB $(0,1.000,2.000$ e $4.000 \mathrm{mg} / \mathrm{l})$.

Figure 4 - Height average of microcuttings and minicuttings of four Eucalyptus grandis clones at 50 day of age (ALT50), in response to IBA application (0, 1000, 2000 and $4000 \mathrm{mg} / \mathrm{l})$. 

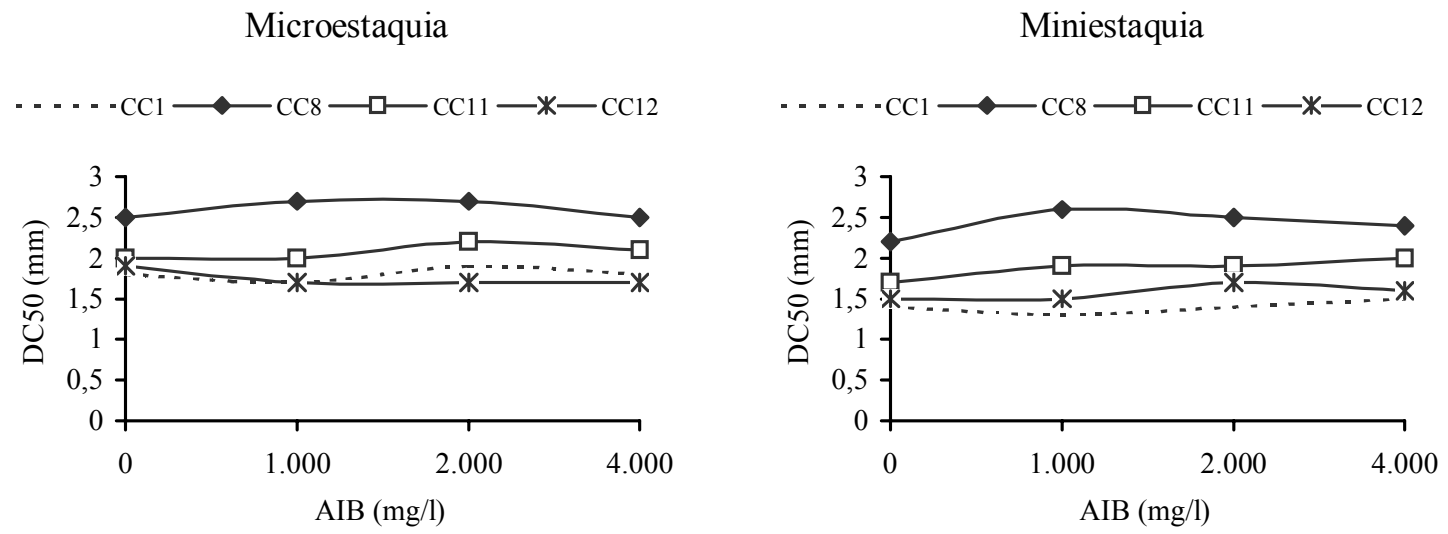

Figura 5 - Médias do diâmetro do colo das mudas de quatro clones de Eucalyptus grandis aos 50 dias de idade (DC50), em resposta à aplicação de $\mathrm{AIB}(0,1.000,2.000$ e $4.000 \mathrm{mg} / \mathrm{l})$.

Figure 5 - Root collar diameter average of microcuttings and minicuttings of four Eucalyptus grandis clones at 50 days of age (DC50), in response to IBA application (0, 1000, 2000 and $4000 \mathrm{mg} / \mathrm{l})$.

técnicas, que os clones apresentaram crescimento diferenciado em altura e em diâmetro do colo, no entanto, dentro de cada clone, não foram observadas diferenças entre as dosagens de AIB. Esses resultados evidenciam que as dosagens de AIB utilizadas não tiveram influência no crescimento das mudas, com a ressalva de que esse parâmetro, de acordo com Carneiro (1995), pode ser facilmente modificado conforme o manejo empregado no processo de produção da muda.

\section{CONCLUSÕES}

A aplicação de 1.000 a $2.000 \mathrm{mg} / \mathrm{l}$ de AIB proporcionou os melhores índices de enraizamento e sobrevivência de miniestacas, na maioria dos clones estudados. Entretanto, para a microestaquia a não-aplicação do AIB proporcionou respostas iguais ou superiores às obtidas na miniestaquia, o que indica maior potencial de enraizamento das microestacas em relação às miniestacas, provavelmente decorrente do rejuvenescimento obtido pela micropropagação.

\section{AGRADECIMENTO}

À Empresa Celulose Nipo-Brasileira S.A. (CENIBRA), pela oportunidade da realização do presente trabalho nas dependências do viveiro florestal e pela disponibilização do material genético (clones); e à CAPES (Coordenadoria de Aperfeiçoamento de Pessoal de Ensino Superior), pelo suporte financeiro.

\section{REFERÊNCIAS BIBLIOGRÁFICAS}

ASSIS, T. F. Propagação vegetativa de Eucalyptus por microestaquia. In: IUFRO CONFERENCE ON SILVICULTURE AND IMPROVEMENT OF EUCALYPTS, 1997, Salvador. Proceedings... Colombo: EMBRAPA, 1997. v. 1. p. 300-304.

ASSIS, T. F.; ROSA, O. P.; GONÇALVES, S. I. Propagação por microestaquia. In: CONGRESSO FLORESTAL ESTADUAL, 7., 1992, Nova Prata. Anais... Santa Maria: UFSM, 1992. p. 824-836.

BLAZICH, F. A. Chemicals and formulations used to promote adventitious rooting. In: DAVIES, T. D.; HAISSIG, B. E.; SANKHLA, N. (Eds.). Adventitious root formation in cuttings. Portland: Dioscorides Press, 1987. p. 132-149. (Advances in Plant Sciences Series, 2).

CARNEIRO, J. G. A. Produção e controle de qualidade de mudas florestais. Curitiba: UFPR/FUPEF, 1995. $451 \mathrm{p}$.

CHUNG, D. Y.; LEE, K. J. Effects of clones, ortet age, crown position, and rooting substance upon the rooting of cuttings of Japanese larch (Larix leptolepis S. et Z. Gordon). Forestry Genetics Research Institute, v. 83, n. 2, p. 205210, 1994. (CD-ROM)

GOMES, A. L. Propagação clonal: princípios e particularidades. Vila Real: Universidade de Trás-osMontes e Alto Douro, 1987. 69 p. (Série Didáctica, Ciências Aplicadas, 1). 
HARTMANN, H. T. et al. Plant propagation: principles and practices. 6.ed. New Jersey: Prentice-Hall, 1997. $770 \mathrm{p}$.

KAMLESH, K. et al. Effect of auxins and carbendazim on rooting of juvenile and mature stem cuttings of Grewia optiva. Indian Journal of Forestry, v. 18, n. 1, p. 61-65, 1995. (CD-ROM)

TITON, M. Propagação clonal de Eucalyptus grandis por miniestaquia e microestaquia. 2001. 65 f. Dissertação (Mestrado em Ciência Florestal) - Universidade Federal de Viçosa, Viçosa-MG, 2001.

WENDLING, I. Propagação clonal de híbridos de Eucalyptus spp. por miniestaquia. 1999. 70 f. Dissertação (Mestrado em Ciência Florestal) - Universidade Federal de Viçosa, Viçosa-MG, 1999.

WENDLING, I. et al. Propagação clonal de híbridos de Eucalyptus spp. por miniestaquia. Revista Árvore, v. 24, n. 2, p. 181-186, 2000a.
WENDLING, I. et al. Efeito do regulador de crescimento AIB na propagação de clones de Eucalyptus spp. por miniestaquia. Revista Árvore, v. 24, n. 2, p. 187-192, 2000b.

WILSON, P. J. Contributions of the leaves and axillary shoots to rooting in Eucalyptus grandis W. Hill ex Mayden. stem cuttings. Journal of Horticultural Science, v. 69, n. 6, p. 999-1007, 1994a.

WILSON, P. J. The concept of a limiting rooting morphogen in woody stem cuttings. Journal of Horticultural Science, v. 9, n. 4, p. 391-400, 1994 b.

XAVIER, A.; COMÉRIO, J. Microestaquia: uma maximização da micropropagação de Eucalyptus. Revista Árvore, v. 20, n. 1, p. 9-16, 1996.

XAVIER, A.; WENDLING, I. Miniestaquia na clonagem de Eucalyptus. Viçosa, MG: SIF, 1998. 10 p. (Informativo Técnico SIF, 11). 